\title{
Conventional parathyroidectomy versus noninstrumental minimally invasive parathyroidectomy in parathyroid adenoma
}

\author{
Mustafa ALIMOĞULLARI ${ }^{1}$, Hakan BULUŞ¹ \\ ${ }^{1}$ Department of General Surgery, Keçiören Training and Research Hospital, Ankara, Turkey \\ Correspondence \\ Mustafa ALIMOĞULLARI \\ Keçiören Eğitim ve Araştırma Hastanesi, Genel Cerrahi Bölümü, Ankara, Türkiye \\ e-mail: alimogullari49@gmail.com
}

\begin{abstract}
In recent years, minimally invasive parathyroidectomy (MIP) is frequently preferred in treating primary hyperparathyroidism (PHPT). The aim of this study was to compare the results obtained using MIP with no instrument and those after conventional parathyroidectomy $(\mathrm{CP})$ in patients with single parathyroid adenoma (SA).

Patients who underwent surgery with SA diagnosis between January 2010 and June 2017 were analyzed retrospectively. Patients with the preoperative multiglandular disease were not included in the study. The patients with SA diagnosis were divided into two groups according to the type of surgery they underwent: MIP and CP. Their demographic characteristics; duration of surgery; preoperative and postoperative parathormone $(\mathrm{PTH})$, calcium $(\mathrm{Ca})$, phosphorus $(\mathrm{P})$, and alkaline phosphatase (ALP) values; localization study results; and pathological diagnosis were compared.

A total of 74 patients were operated for SA. Further, 42 (56.7\%) of the patients were operated with the MIP technique and 32 (43.2\%) with the CP technique. The mean age of the patients was $52.0 \pm 12$ years for MIP and was $56.7 \pm 12$ years for CP. The mean operative time was $66.1 \pm 27.3 \mathrm{~min}$ for MIP and $106.5 \pm 44.6 \mathrm{~min}$ for CP. The success rate was $92.8 \%$ for MIP and $100 \%$ for CP. Neck ultrasonography (USG) and parathyroid scintigraphy (PS) were performed on all patients for preoperative diagnosis and localization. The accuracy rates were $66.2 \%$ for USG and $89.1 \%$ for PS. No significant difference was found between USG and PS sensitivity with regards to adenoma diameter. The mean follow-up period was $13.2 \pm 9.7$ months (range 3-32 months).

MIP is an appropriate surgical choice in SA treatment if preoperative localization studies are performed at a proper and adequate level. In MIP, the success rate of the surgery increases significantly in parallel with the increase in the performing team's experience.
\end{abstract}

Key words: Conventional parathyroidectomy, minimally invasive parathyroidectomy, parathyroid adenoma

\section{INTRODUCTION}

Parathyroid glands are usually found with intrathyroidal involvement behind the thyroid glands and are responsible for parathyroid hormone (PTH) secretion. Rarely, they can also be localized as extrathyroidal with thyroid agenesis [1].

Primary hyperparathyroidism (PHPT) is a common endocrine system disease characterized by the excessive release of PTH. PHPT is the third most common endocrine disorder in the United States with an annual incidence of 100,000 [2]; 1 out of every 500 women and 1 out of every 2000 men are affected by this disease annually [3]. A quick review of the literature shows that around $80 \%$ of PHPT cases are single parathyroid adenoma (SA), 15\% are parathyroid hyperplasia, $5 \%$ are multiple parathyroid adenoma, and $1 \%$ are parathyroid cancer [4].
PHPT is usually asymptomatic. Symptomatic PHPT has various symptoms of hypercalcemia, such as fatigue, nausea, loss of appetite, peptic ulcer disease, diarrhea, changes in mental state, recurrent kidney stones, and bone disease or bone loss [5].

The conventional method of choice for parathyroidectomy is bilateral neck exploration (BNE). In BNE, bilateral exploration is performed, and gland/glands thought to be pathological are excised. BNE was considered the gold standard surgery; after the introduction of parathyroid scintigraphy (PS), unilateral neck exploration and selective parathyroidectomy (SP) started to gain popularity. SP can be performed as open, minimally invasive parathyroidectomy (MIP), endoscopic, radio-guided, or video-assisted [6]. 
In the MIP procedure, which is commonly used today, care should be taken to ensure that PHPT is due to $\mathrm{SA}$ and preoperative localization workup is performed very well. Despite a similar success rate and recurrence rates, MIP has lower cost and morbidity rates, making it more preferable over BNE [7]. Rapid serum PTH (rPTH) or gamma probe can be used intraoperatively to increase MIP success rate. The operation is regarded successful if the intraoperative rPTH level after parathyroid adenoma excision decreases more than $50 \%$ of the preoperative PTH level. However, MIP operation can be performed successfully without any instrumentation due to the absence of rPTH measurement and gamma probe in many centers.

Neck ultrasonography (USG) and PS (usually both) are used for preoperative diagnosis. Advanced diagnostic methods such as computed tomography (CT), magnetic resonance imaging (MRI), and SPECTCT are rarely needed [8].

This study aimed to evaluate the results of patients with SA operated with the noninstrumental MIP technique or conventional parathyroidectomy (CP).

\section{MATERIALS AND METHODS}

TA total of 74 patients with a preoperative SA diagnosis, who underwent surgery between January 2010 and June 2017 at the clinic, were evaluated retrospectively. Patients with a diagnosis of multiglandular parathyroid adenoma (MGD) and parathyroid carcinoma were excluded from the study. The demographic characteristics; duration of surgery; preoperative and postoperative (24th hour) PTH, calcium (Ca), phosphorus $(P)$, and alkaline phosphatase (ALP) values; results of the preoperative diagnostic evaluation with US, PS, $\mathrm{CT}$, and MRI; the size and weight of pathological specimens; and the postoperative follow-up period of the patients were assessed. The bone mineral density and vitamin $D$ levels were not added to the study because they were not recorded for all patients.

In these patients with hypercalcemia and elevated PTH levels, neck USG and PS were used to detect parathyroid pathologies. As USG can detect accompanying thyroid pathologies, it was used on all patients. In PS, double phase images were taken 15 min (early phase) and $2 \mathrm{~h}$ (late phase) following the intravenous injection of $25 \mathrm{mCl}$ Technetium-99m. Focal activity involvement in early and late phases was accepted as positive pathological PS.
$\mathrm{CP}$ was performed by excising the pathological gland after the exploration and visualization of all parathyroid glands bilaterally with a 6-cm incision under general anesthesia. On the contrary, MIP was performed on 3 patients under local anesthesia and 39 patients under general anesthesia, at a preoperatively marked location with a $1.5-$ to $2-\mathrm{cm}$ incision directly above SA or a midline 2-cm mini-incision. In both procedures, additional adjunctive methods such as gamma probe or intraoperative rPTH were not used, and the excised specimen was sent for the frozen-section study during the surgery to confirm the pathology and end the surgery. The blood $\mathrm{Ca}, \mathrm{PTH}$, and P values were obtained in both surgical groups $24 \mathrm{~h}$ before and after the surgery. The MIP group was discharged on the first day and the CP group on the second day after the surgery.

Recurrence was defined as presenting with elevated Ca and PTH levels 6 months after the surgery. Persistence was defined as elevated $\mathrm{Ca}$ and PTH within 6 months after the surgery [9].

Data were analyzed using SPSS for Windows v.15.0 (SPSS, Inc., IL, USA). Descriptive and frequency analytics were performed. The $t$ test was used for analyzing the samples. The statistical significance level was set as $P$ less than 0.05 .

\section{RESULTS}

A Between January 2010 and June 2017, a total of 74 patients with SA diagnosis were operated in the clinic; $42(56.7 \%)$ of the patients were operated with the MIP technique and 32 (43.2\%) with the CP technique. All of the CP patients had concomitant thyroid pathologies, and MIP was not performed because of concomitant thyroidectomy. Further, 13 (40.6\%) of 32 patients in the CP group underwent unilateral lobectomy, and 19 (59.4\%) underwent bilateral total thyroidectomy (Table 1).

For the preoperative diagnosis and localization, USG and PS images were obtained from all of the patients. Neck MRI $(n=12,16.2 \%)$ and/or neck CT $(n=$ $5,6.7 \%)$ were used in patients with uncertain adenoma localization. The accuracy rates were found to be $66.2 \%$ for USG, $89.1 \%$ for PS, $41.6 \%$ for MRI, and $40 \%$ for CT (Table 2). No statistically significant difference was found between adenoma diameter and USG sensitivity $(P=0.385)$ or PS sensitivity $(P=0.829)$. 
Table 1: Clinical characteristics and postoperative comparison of patients.

\begin{tabular}{llll}
\hline & MIP & CP & P value \\
\hline Number & 42 & 32 & 0.106 \\
Age & $52.0 \pm 12$ & $56.7 \pm 12$ & 0.494 \\
Sex (men/women) & $8 / 34$ & $7 / 25$ & \\
Thyroidectomy (Lobectomy/BTT) & 0 & $13 / 19$ & $<0.001$ \\
Surgical time (min) & $66.1 \pm 27.3$ & $106.5 \pm 44.6$ & 0.494 \\
Complications (wound inf., hematoma, and hypocalcemia) & 5 & 7 & 0.177 \\
Persistent hyperparathyroidism & 3 & 0 & 0.482 \\
Adenoma weight (g) & $1.48 \pm 0.46$ & $1.58 \pm 0.76$ & 0.502 \\
Adenoma size (mm) & $18.14 \pm 5.60$ & $19.9 \pm 9.73$ & 2 \\
\hline
\end{tabular}

BTT: Bilateral total thyroidectomy CP: Conventional parathyroidectomy MIP: Minimally invasive parathyroidectomy

The mean age of the patients was $56.7 \pm 1.4$ ( 25 74) years in the $C P$ group and $52.0 \pm 1.3(29-74)$ years in the MIP group $(P=0.106)$. A total of $59(79.7 \%)$ patients were female (MIP: 34; CP: 25), and 15 (20.2\%) were male (MIP: $8 ; \mathrm{CP}: 7)(P=0.494)$ (Table 1). No statistically significant difference was found between the two groups in terms of preoperative and postoperative PTH (Fig. 1), Ca (Fig. 2), phosphorus, and ALP values (Table 3 ). The only statistically significant difference between the two groups was found out to be in the surgical times. The surgical time of MIP and CP was $66.1 \pm 27.3 \mathrm{~min}$ and $106.5 \pm 44.6 \mathrm{~min}(P<0.001)$, respectively (Table 1$)$. However, additional thyroidectomy was thought to have an effect on prolonging the duration of $C P$.

The frozen results of the specimens taken in all surgeries were reported as parathyroid adenoma. The comparisons between adenoma diameter or weight and elevated PTH or Ca levels yielded no statistically significant results either.

Parathyroid adenoma was most commonly located at the right lower quadrant in operated pa- tients (Table 4). The adenoma diameter in the CP group was $19.9 \pm 9.73 \mathrm{~mm}$ and the weight was 1.58 $\pm 0.76 \mathrm{~g}$; they were $18.14 \pm 5.60 \mathrm{~mm}$ and $1.48 \pm 0.46$ $\mathrm{g}$, respectively, in the MIP group $(P=0.482)$ (Table 1$)$.

The mean follow-up period was $13.2 \pm 9.7$ months (ranging between 3 and 32 months). The postoperative complications were hematoma, wound infection, hypocalcemia, and, most importantly, persistent hyperparathyroidism. No nerve damage or recurrent hyperparathyroidism was observed in the patients. Although complications were less in the MIP group, no statistically significant difference was found between groups in this regard. Considering the success rates of the techniques, persistent hyperparathyroidism was detected in three patients in the MIP group (success $92.8 \%$ ), but none in the CP group (success $100 \%)$. However, this difference was not statistically significant $(P=0.177$ ) (Table 1). All three patients with recurrence in the MIP group underwent surgery in the earlier stages of adopting MIP in the clinic (in 2010 and 2011), and the success rate increased with increased experience in preoperative imaging and

Table 2: Preoperative localization studies.

\begin{tabular}{llll}
\hline Imaging method & $\mathrm{N}(\%)$ & Accuracy rate for localization (\%) & Accuracy rate for lateralization (\%) \\
\hline US & $74(100)$ & 66.2 & 66.2 \\
PS & $74(100)$ & 89.1 & 91.8 \\
MRI & $12(16.2)$ & 40 & 40 \\
CT & $5(6.7)$ & 41.6 & 41.6 \\
\hline
\end{tabular}

CT: Computed tomography MRI: Magnetic resonance imaging PS: Parathyroid scintigraphy US: Ultrasound. 


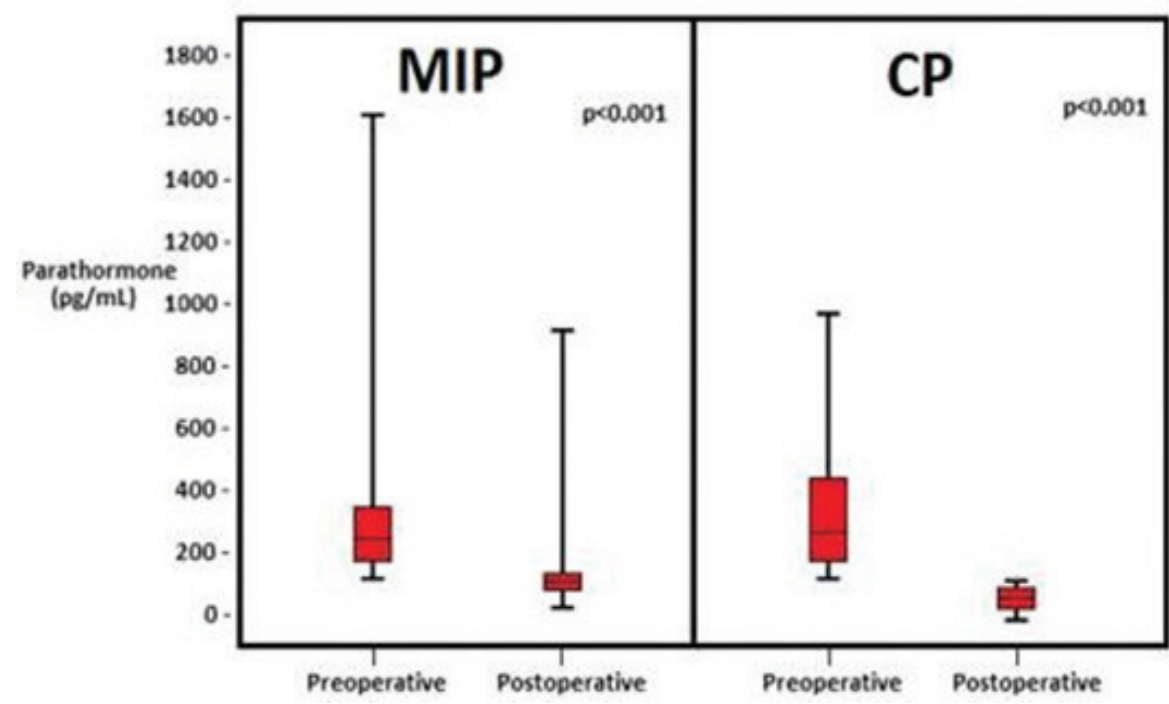

Figure 1: Parathormone levels of patients measured before the surgery and $24 \mathrm{~h}$ after the surgery in the MIP and CP groups.

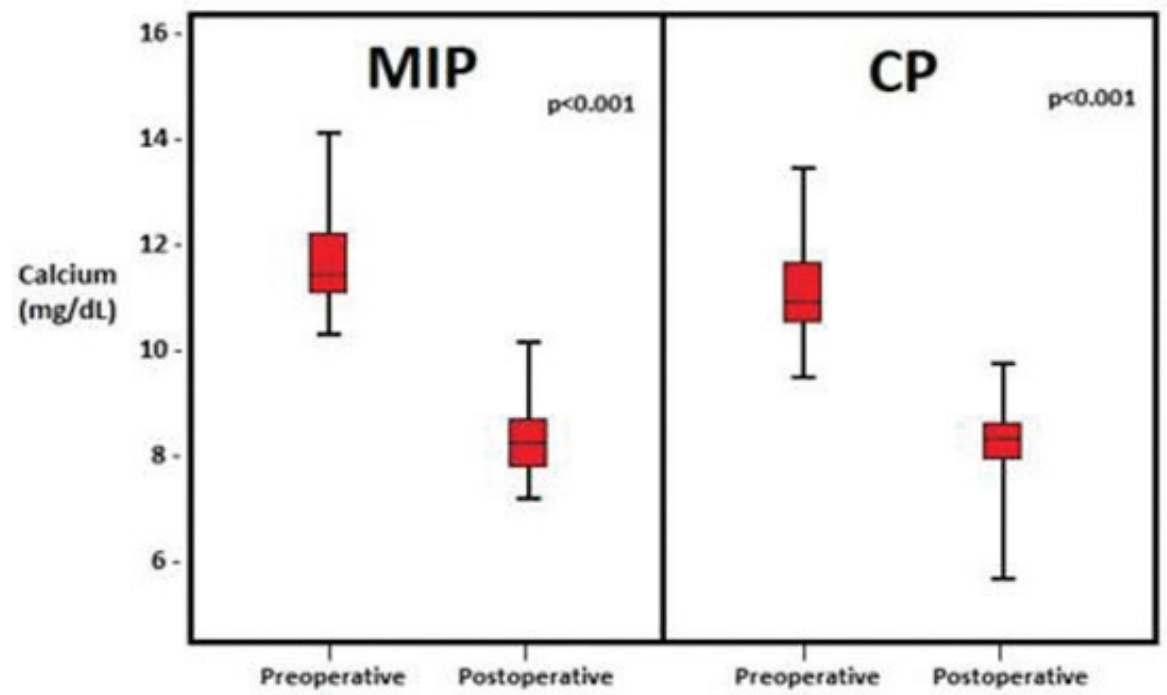

Figure 2: Calcium levels of patients measured before the surgery and $24 \mathrm{~h}$ after the surgery in the MIP and CP groups.

surgery. In addition, the mean operative time in the MIP group was shortened in parallel with the surgical learning curve over the years (Fig. 3).

\section{DISCUSSION}

The definitive therapeutic method in PHPT is parathyroidectomy. BNE, which was first introduced in 1925 and accepted as the CP method, was performed to visualize and excise the pathological gland or glands [10]; the success rates ranged from 90 to $95 \%$ [11]. However, with the introduction of PS in 1992 and the increase in USG quality over the years, the preoperative localization of parathyroid adenoma can be detected more clearly. As a result, interventions such as MIP aimed at the excision of a pathological gland became more popular only in recent years. Smaller incision scar, shorter surgical time, less postoperative pain, shorter hospital stay, and lower cost are the major advantages of MIP [2]. Postoperative complications such as nerve damage, postoperative hemorrhage, wound infection, and hypoparathyroidism are less frequent [12]. Although complications were less in the MIP group, no statistically significant difference was found between the groups in this regard $(P=0.494)$. In addition, many studies showed that the postoperative quality of life 
Table 3: Laboratory values of patients.

\begin{tabular}{llll}
\hline & MIP & CP & P value \\
\hline Preop PTH & $302.33 \pm 319.9$ & $308.73 \pm 235.3$ & 0.925 \\
Postop PTH & $38.13 \pm 28.7$ & $26.93 \pm 26.9$ & 0.092 \\
Preop Ca & $11.53 \pm 0.85$ & $11.23 \pm 0.93$ & 0.309 \\
Postop Ca & $9.23 \pm 0.82$ & $8.93 \pm 0.98$ & 0.116 \\
Preop P & $2.43 \pm 0.51$ & $2.43 \pm 0.65$ & 0.858 \\
Postop P & $2.73 \pm 0.81$ & $2.93 \pm 0.97$ & 0.532 \\
Preop ALP & $136.73 \pm 93.8$ & $142.83 \pm 67.5$ & 0.755 \\
Postop ALP & $121.13 \pm 131.4$ & $114.53 \pm 54.8$ & 0.793 \\
\hline
\end{tabular}

ALP: Alkaline phospatase Ca: Calcium P: Phosporu PTH: Parathormone

Normal Intervals, $\mathrm{PTH}=22.2-108.9 \mathrm{pg} / \mathrm{mL}, \mathrm{Ca}=8.8-10.6 \mathrm{mg} / \mathrm{dL}, \mathrm{P}=2.5-4.5 \mathrm{mg} / \mathrm{dL}, \mathrm{ALP}=30-120 \mathrm{IU} / \mathrm{L}$.

Table 4: Adenoma localization.

\begin{tabular}{ll}
\hline & Number/Percentage \\
Right inferior & $37(50)$ \\
Right superior & $4(5.4)$ \\
Left inferior & $30(40.5)$ \\
Left superior & $3(4.1)$ \\
\hline
\end{tabular}

was better in patients undergoing MIP [13].

The most important factor for MIP success is establishing the correct adenoma localization. It is very important to distinguish the MGD from SA before the surgery. Today, the methods used in preoperative localization studies are USG and PS. In addition, methods such as neck CT, MRI, and SPECT-CT can be used $[8,14]$. Many studies have suggested that the first tests to be performed for diagnosis are the PS and USG combination $[8,15]$. Ultrasound should be done

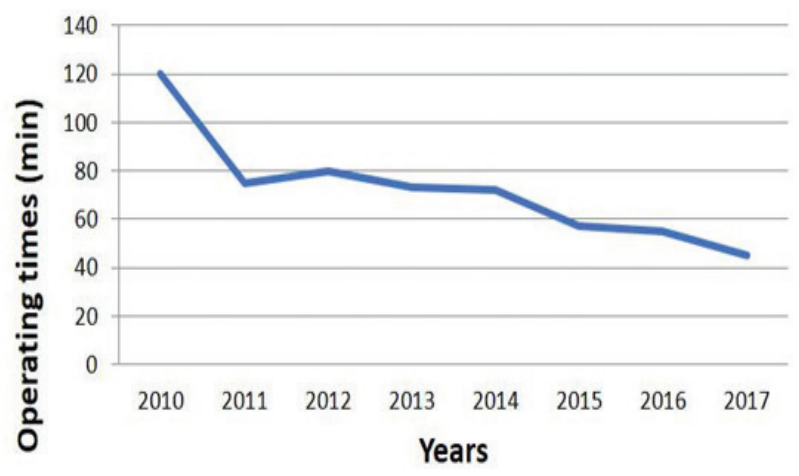

Figure 3: Median surgical times of MIP over the years. because it can show the probable accompanying thyroid pathologies. The diagnosis rate for USG and PS was $40 \%-99 \%$ and $91 \%-98 \%$, respectively [14]. The diagnostic rate of USG was significantly reduced in PS-negative patients; the rate was determined to be $42 \%$ in a large-scale cohort study [16]. When PS and USG were combined, the diagnostic rate increased to $95 \%$. The diagnostic rates for CT and MRI were between $50 \%$ and $60 \%$ [14].

In the present study, the diagnostic rate was $66.2 \%$ for USG and $91.8 \%$ for PS; it was found to be $95.9 \%$ with the combination of the two techniques, which was consistent with previous findings. Despite an increase in the sensitivity of PS and USG with the increase in the diameter of adenoma, no statistically significant correlation between was observed between adenoma diameter and USG or PS sensitivity in the present study. The diagnostic rate for MRI and CT was found to be $40 \%$ and $41.6 \%$, respectively, but they were not significant because the assessments were performed on a small number of patients. In two patients, when MGD was present, USGs were negative and PS was reported as solitary parathyroid adenoma. Since these two patients had MIP, hyperparathyroidism persisted postoperatively, leading to reoperation. This proved that MIP success was directly related to preoperative diagnostic methods. In accordance with previous findings, it is believed that $\mathrm{CP}$ should be preferred over MIP in patients with uncertain preoperative diagnosis.

Instruments such as rPTH measurement and/or gamma probe can be used to increase the MIP success rate. However, the use of these instruments remains controversial because they can increase costs, 
prolong surgical duration, and yield false-negative or false-positive results. Some studies showed that the rate of success increased to $100 \%$, thus supporting the use of these instruments [16-19]. However, many studies proved that the success rate did not improve and hence the instruments were not cost-effective [20-22]. Non-routine use in some studies suggested that the instruments should be used only in patients with uncertain preoperative adenoma localization or recurrent adenomas [23]. Considering that these instruments increased the cost and had a minimal contribution to success, they were not used in the present study.

The present study had certain limitations. It had the errors and biases inherent in a retrospective study design. Another limitation was the small sample size. Large-sample studies might help in better analysis.

MIP should be preferred over CP in treating patients with SA because the complication risk is low, surgical time is short, and the costs and morbidity rates are lower. The use of instruments such as rPTH and gamma probe to increase surgical success in MIP is controversial. However, the most important factor for the success of MIP is correct preoperative localization. The success of MIP decreases for patients with MGD or patients with incorrect preoperative localization.

\section{Conflicts of Interest and Ethics Statement}

Ethical approval for the present study was not required because it was retrospectively performed. The authors declare no conflicts of interest-financial or otherwise-related to the material presented herein.

\section{REFERENCES}

1. Akkurt G, Bulus $\mathrm{H}$, Atas $\mathrm{H}$ et al. Bilobar Thyroid Agenesis: Rare Case. Turkiye Klinikleri J Endocrin 2016;11(2):65-8

2. Adler JT, Sippel RS, Chen H. New trends in parathyroid surgery. Curr Probl Surg 2010;47:9581017

3. Bilezikian JP, Silverberg SJ. Clinical spectrum of primary hyperparathyroidism. Rev Endocr Metab Disord 2000; 1: $237-$ 245.

4. E. Hindie, O. Ugur, D. Fuster, M. O'Doherty, G. Grassetto, P. Urena, et al.,Parathyroid guidelines, Eur. J. Nucl. Med. Mol. Imaging 36 (2009) 1201-1216.

5. L.J. Melton, Epidemiology of primary hyperparathyroidism, J. Bone Min Res 6 (1991) S25-S30

6. Grant CS, Thompson G, Farley D, van Heerden J. Primary hyperparathyroidism surgical management since the introduction of minimally invasive parathyroidectomy: Mayo Clinic experience. Arch Surg 2005; 140: 472-478.

7. Woong Kim et al. Is focused parathyroidectomy appropriate for patients with primary hyperparathyroidism? Ann Surg Treat Res 2016;91(3):97-103
8. Gross ND, Weissman JL, Veenker E, Cohen JI. The diagnostic utility of computed tomography for preoperative localization in surgery for hyperparathyroidism. Laryngoscope 2004;114:227231.

9. Wirowski D, Goretzki PE, Schwarz K, Lammers BJ, Dotzenrath C, Roher HD. Failed surgery in primary hyperparathyroidism: what has changed with time. Exp Clin Endocrinol Diabetes 2013;121:3238

10. Palazzo F, Sadler GP. Minimally invasive parathyroidectomy, heralds a new era in the treatment of primary hyperparathyroidism. BMJ 2004; 328: 849-850.

11. Allendorf J, DiGorgi M, Spanknebel K, Inabnet W, ChabotJ, Logerfo P. 1112 consecutive bilateral neck explorations for primary hyperparathyroidism. World J Surg 2007;31:20752080.

12. Karakas E, Schneider R, Rothmund M, Bartsch DK, Schlosser $\mathrm{K}$. Initial surgery for benign primary hyperparathyroidism: an analysis of 1,300 patients in a teaching hospital. World J Surg 2014;38:2011-2018.

13. Tjun T, Seamus D, Bruce R, Leigh D. Does the surgical approach affect quality of life outcomes? A comparison of minimally invasive parathyroidectomy with open parathyroidectomy. Int J Surg 2007; 5,17-22

14. Noda S, Onoda N, Kashiwagi H, Kawajiri H, Takashima T, Ishikawa T et al. Strategy of operative treatment of hyperparathyroidism using US scan and Tc-99m-MIBI SPECT/CT. Endocr J 2014. 61:225-230

15. Korukluoğlu B, Kıyak G, Çelik A, Ucar AE, Ergül E, Kuşdemir A. Our surgical approach to parathyroid adenoma and the role of localization studies. Türkiye Klinikleri J Med Sci 2008; 28: 24-29.

16. D.M. Elaraj, R.S. Sippel, S. Lindsay, I. Sansano, Q.Y. Duh, O.H. Clark, et al., Are additional localization studies and referral indicated for patients with primary hyperparathyroidism who have negative sestamibi scan results? Arch Surg 145 (2010) 578-581.

17. Vignali $E$, Picone A, Materazzi G, Steffe $S$, Berti P, Cianferotti $L$, et al. A quick intraoperative parathyroid hormone assay in the surgical management of patients with primary hyperparathyroidism: a study of 206 consecutive cases. Eur J Endocrinol 2002; 146: 783-788

18. Proctor MD, Sofferman RA. Intraoperative parathyroid hormone testing: what have we learned? Laryngoscope 2003; 113: 706-714.

19. Burkey SH, Van Heerden JA, Farley DR, Thompson GB, Grant $\mathrm{CS}$, Curlee KJ. Will directed parathyroidectomy utilizing the gamma probe or intraoperative parathyroid hormone assay replace bilateral cervical exploration as the preferred operation for primary hyperparathyroidism? World J Surg 2002; 26: 914920.

20. Weber CJ, Ritchie JC. Retrospective analysis of sequential chang-es in serum intact parathyroid hormone levels during conven-tional parathyroid exploration. Surgery 1999; 126: 1139-1143.

21. Stalberg P, SidhuS, SywakM, Robinson B, Wilkinson M, Delbridge L. Intraoperative parathyroid hormone measurement during minimaly invasive parathyroidectomy: Does it "Value-add" to decision-making? J Am Coll Surg 2006; 203: 1-6.

22. Alireza N, Stacie K, Matthew TO, Ralph PT, Martha AZ. Intraoperative PTH May Not Be Necessary in the Management of Primary Hyperparathyroidism Even with Only One Positive or Only Indeterminate Preoperative Localization Studies World J Surg 10.1007/s00268-017-3871-4

23. Garner SC, Leight GS Jr. Initial experience with intraoperative PTH determinations in the surgical management of 130 consecutive cases of primary hyperparathyroidism. Surgery 1999; 126: 1132-1137. 